\title{
Bifurcation to heteroclinic cycles and sensitivity in three and four coupled phase oscillators
}

\author{
Peter Ashwin \\ Mathematics Research Institute, \\ School of Engineering, Computer Science and Mathematics, \\ University of Exeter, Exeter EX4 4QE, UK \\ Oleksandr Burylko \\ Institute of Mathematics, National Academy of Sciences of \\ Ukraine, 01601 Kyiv, Ukraine \\ Yuri Maistrenko, \\ Institute of Medicine and Virtual Institute of Neuromodulation, \\ Research Centre Jülich, 52425 Jülich, Germany; \\ Institute of Mathematics, National Academy of Sciences of \\ Ukraine, 01601 Kyiv, Ukraine
}

July 17, 2007

\begin{abstract}
We study the bifurcation and dynamical behaviour of the system of $N$ globally coupled identical phase oscillators introduced by Hansel, Mato and Meunier, in the cases $N=3$ and $N=4$. This model has been found to exhibit robust 'slow switching' oscillations that are caused by the presence of robust heteroclinic attractors. This paper presents a bifurcation analysis of the system in an attempt to better understand the creation of such attractors. We consider bifurcations that occur in a system of identical oscillators on varying parameters in the coupling function. These bifurcations preserve the permutation symmetry of the system. We then investigate implications of these bifurcations for the sensitivity to detuning (i.e. the size of the smallest perturbations that give rise to loss of frequency locking).

For $N=3$ we find three types of heteroclinic bifurcation that are codimensionone with symmetry. On varying two parameters in the coupling function we find three curves giving (a) an $S_{3}$-transcritical homoclinic bifurcation, (b) a saddle-node/heteroclinic bifurcation and (c) a $\mathbb{Z}_{3}$-heteroclinic bifurcation. We also identify several global bifurcations with symmetry that organize the bifurcation diagram; these are codimension-two with symmetry.
\end{abstract}


For $N=4$ oscillators we determine many (but not all) codimension-one bifurcations with symmetry, including those that lead to a robust heteroclinic cycle. A robust heteroclinic cycle is stable in an open region of parameter space and unstable in another open region. Furthermore, we verify that there is a subregion where the heteroclinic cycle is the only attractor of the system, while for other parts of the phase plane it can coexist with stable limit cycles. We finish with a discussion of bifurcations that appear for this coupling function and general $N$, as well as for more general coupling functions.

\section{Introduction}

Phase oscillator systems of the form

$$
\dot{\theta}_{i}=\omega_{i}+\frac{1}{N} \sum_{j=1}^{N} g\left(\theta_{i}-\theta_{j}\right)
$$

arise quite naturally as descriptions of weakly coupled limit cycle oscillators, where $\omega_{i}$ represents the natural frequency of oscillator $i$ and the coupling function $g$ represents the interaction between oscillators. Such systems of globally coupled oscillators are of great interest not only because of their applications in physics and biology [28, 26, 9], but also because they provide examples of how systems with simple dynamics can interact to give highly nontrivial collective dynamics [24]. Since the work of Winfree and Kuramoto [29, 19] there has been much progress in understanding general features of, for example, the onset of various types of synchronization in globally coupled phase oscillator systems.

A particular model is that of Kuramoto [19], where $g(x)=-K \sin (x)$ and $K$ is the coupling strength. In this paper we consider a generalization of the Kuramoto model [20, 24] by Hansel, Mato, Meunier [13, 14, 17, 18] that uses a more general coupling, with the result that some bifurcation degeneracies of the Kuramoto model are removed. The latter model was originally derived as an approximation of coupled neural oscillators [13] and is notable in that it can produce non-trivial clustering dynamics even if the oscillators are identical. This clustering can appear as 'slow switching' for the identical oscillators system where, in the presence of noise or imperfections, the dynamics shows an approximately periodic oscillation between cluster states. Such dynamics has recenlty been observed in a chemical reactor system [16].

This paper aims to understand the generic bifurcations in this model for $N=3$ and 4, and particularly those that give rise to 'slow switching' attractors that are attracting heteroclinic cycles. We consider some general results for larger $N$ in the discussion. We do not consider the case $N=2$ because this cannot give rise to heteroclinic cycles in our model. We find a number of new mechanisms that give rise to the appearance of heteroclinic cycles. Our results indicate that heteroclinic cycles can only be found at codimension-one for $N=3$ but can be robust (exist in open regions in parameter space for identical oscillator systems) for $N=4$ and higher. 
Most of this paper details the behaviour of a particular case of the system (1) for identical oscillators in the absence of detuning, i.e. such that $\omega_{i}=\omega$ independent of $i$. In such cases the 'slow switching' can be explained as robust heteroclinic attractors that, in the presence of noise, exhibit approximately periodic oscillations between dynamically unstable (saddle) states with a period that becomes unbounded as the noise is reduced to zero. This slow switching has been observed in certain types of neural dynamics $[1,7,15]$ and hence the mechanisms studied here may be of particular interest in such models.

A second motivation for this paper is to better understand cases where there is extreme sensitivity of the attractors for system (1) to detuning [5], i.e. how $\omega_{i}$ arbitrarily close to constant can give attractors that break frequency locking, even for strong coupling.

\subsection{A model for globally coupled phase oscillators}

We consider the system of $i=1, \cdots, N$ coupled phase oscillators

$$
\begin{gathered}
\dot{\theta}_{i}=\omega_{i}+\frac{K}{N} \sum_{j=1}^{N} g\left(\theta_{i}-\theta_{j}\right), \quad \text { where } \\
g(x)=g_{\alpha, r}(x)=-\sin (x-\alpha)+r \sin (2 x),
\end{gathered}
$$

where $\theta_{i} \in[0,2 \pi)$ are phase variables, $\omega_{i}$ are natural frequencies, $K>0$ is a coupling parameter and $g(x)$ is a specific coupling function $[3,9]$. We consider the dynamics and bifurcations of this system for identical oscillators

$$
\omega_{i}=\omega, \text { for } i=1, \cdots, N
$$

on varying the parameters $r \in \mathbb{R}$ and $\alpha \in[0,2 \pi)$ in the coupling function $g_{\alpha, r}(x)$. We note that without generality we can assume $r \geq 0$ because of the time-reversing symmetry $g_{\alpha, r}(x)=-g_{\alpha+\pi,-r}(x)$.

This particular choice of $g(x)$ was introduced in [13] and hence we refer to (2) as the Hansel-Mato-Meunier model $[13,17,18]$. In the case $r=0$ (i.e. $g(x)=-\sin (x-\alpha)$ ) this is the Kuramoto-Sakaguchi model, for which the only bifurcations are at $\alpha= \pm \pi / 2$, and these bifurcations are degenerate.

System $(2,3)$ possesses symmetries given by all permutations $S_{N}$ of the oscillators, and this implies that there are a number of orbits in phase space that are fixed by symmetry [3]; one of these is the in-phase solution

$$
\{(\theta, \cdots, \theta): \theta \in \mathbb{T}\}
$$

and another, the set of antiphase solutions

$$
M^{(N)}=\left\{\left(\theta_{1}, \ldots, \theta_{N}\right): \sum_{j=1}^{N} e^{i \theta_{j}}=0\right\} .
$$

The set $M^{(N)}$ is a union of manifolds of dimension $N-2$ for $N \geq 3$; see Appendix A. For any $\alpha$ it consists of fixed points of the phase differences in the case $r=0$ and contains a union of invariant manifolds for more general $r$. 


\subsection{Reduction to phase differences}

To exploit the phase-shift symmetry of this system one can describe the system dynamics in terms of the dynamics of the phase differences

$$
\phi_{i}=\theta_{1}-\theta_{i+1}, \quad i=1, \cdots, N-1,
$$

thus reducing this $N$-dimensional system to the $(N-1)$-dimensional system

$$
\begin{aligned}
\dot{\phi}_{i}= & \Delta_{i}+r \frac{K}{N}\left[\sin \left(2 \phi_{i}\right)+\sum_{j=1}^{N-1}\left(\sin \left(2 \phi_{j}\right)+\sin \left(2\left(\phi_{j}-\phi_{i}\right)\right)\right)\right]- \\
& -\frac{K}{N}\left[\sin \left(\phi_{i}+\alpha\right)+\sum_{j=1}^{N-1} \sin \left(\phi_{j}-\alpha\right)+\sum_{j=1, j \neq i}^{N-1} \sin \left(\phi_{i}-\phi_{j}+\alpha\right)\right]
\end{aligned}
$$

where $\Delta_{i}=\omega_{1}-\omega_{i+1}, i=1, \cdots, N-1$ are the set of detunings of the oscillators. Thus (2) with $N=2$ can be reduced to a scalar equation as described in [5].

We mostly consider the case where $\omega_{i}$ are all equal (3); this condition implies the invariance of the sets

$$
P_{i j}=\left\{\left(\theta_{1} \cdots, \theta_{n}\right): \theta_{i}=\theta_{j}\right\}
$$

for any $i \neq j=\{1, \cdots, N\} .{ }^{1}$ Without loss of generality we can set $K=N$ by scaling time.

\subsection{Sensitivity to detuning}

In real applications the assumption (3) will be broken by imperfections in the system and so it is interesting to ask how far one can perturb (or detune) the $\omega_{i}$ before the oscillators lose frequency synchronization. We say two oscillators $\theta_{i}$ and $\theta_{j}$ have bounded phase difference if $\left|\theta_{j}-\theta_{i}\right|$ is bounded uniformly for $t>0$. Note that if $\theta_{i}$ and $\theta_{j}$ have bounded phase difference then they will be frequency synchronized. For the standard Kuramoto model $(r=\alpha=0)$ the sensitivity increases with decreasing coupling strength $K$. By contrast, for (2) extremely small detuning may result in loss of frequency synchronization even if the coupling is significant [5]. Note that when (3) is not satisfied, it possible for highly complicated dynamics may appear [24, 22]. More precisely we define as in [5] the sensitivity to detuning to be

$$
\Omega=\sup \{\delta:|\Delta|<\delta \text { implies all attractors of (1) have bounded phase differences. }\} .
$$

We say a system has extreme sensitivity if $\Omega=0$. Extreme sensitivity generally appears at points of heteroclinic bifurcation and implies that arbitrarily small detuning destroys frequency locking between oscillators. Work in [5] indicates that this can appear robustly for $N \geq 5$ coupled oscillators due to the appearance of robust heteroclinic attractors that when lifted to the torus form a single connected component. In this paper we work on clarifying the appearance of extreme sensitivity for $N=3$ and $N=4$ for a particular system of coupled phase oscillators.

\footnotetext{
${ }^{1}$ Note that this only applies for the case of no detuning.
} 
Topological sensitivity We introduce a concept intimately related to sensitivity, namely topological sensitivity to detuning. Suppose that the set of all attractors of (1) is $\mathcal{A}_{\Delta}$ for given detuning $\Delta_{k}=\omega_{k}-\omega_{n}, \quad k=1, \cdots, n-1$, so that $\mathcal{A}_{0}$ signifies the attractors for the system with (3). We define

$$
\Omega^{\text {top }}=\sup \left\{\delta: B_{\delta}\left(\mathcal{A}_{0}\right) \text { only has components that are contractible to the diagonal }\right\}
$$

where $B_{\delta}\left(\mathcal{A}_{0}\right)$ is the $\delta$-neighbourhood of $\mathcal{A}_{0}$ within $\mathbb{T}^{N}$. If a set $\mathcal{A}_{0} \subset \mathbb{T}^{N}$ only has components that are contractible to the diagonal ${ }^{2}(1, \cdots, 1) \subset \mathbb{T}^{N}$ then it follows that all trajectories attracted to $\mathcal{A}_{0}$ must have bounded phase differences. We use this to characterise extreme sensitivity.

Lemma 1 If all attractors $\mathcal{A}_{0}$ are asymptotically stable and $\Omega=0$ then $\Omega^{\text {top }}=0$.

Proof: We prove by contradiction; namely we show that if all attractors are asymptotically stable and $\Omega^{t o p}>0$ then $\Omega>0$. Note that if all attractors are asymptotically stable then they are upper semicontinuous in the following sense: given any $\eta>0$ there is a $\delta>0$ such that if $\|\Delta\|<\delta$ then $B_{\eta}\left(\mathcal{A}_{0}\right) \supset \mathcal{A}_{\Delta}$; see for example [2]. Now suppose that $\eta=\Omega^{\text {top }}>0$; there is a $\delta>0$ such that for all $\|\Delta\|<\delta$ we have

$$
B_{\eta / 2}\left(\mathcal{A}_{0}\right) \supset \mathcal{A}_{\Delta}
$$

Because $\mathcal{A}_{0}$ is closed and only contains components contractible to the diagonal the same must hold for $B_{\eta / 2}\left(\mathcal{A}_{0}\right)$ with $\eta$ small enough. Hence by choosing an appropriate $\delta>0$, all components of the perturbed attractors $\mathcal{A}_{\Delta}$ with $\|\Delta\|<\delta$ will remain contractible to the diagonal. Hence $\Omega \geq \delta>0$.

QED

Note that the assumption that the attractors are asymptotically stable includes many types of heteroclinic attractor. A converse to Lemma 1 requires more stringent conditions that we have not investigated in detail. However, we can quantify a direct link between heteroclinic cycles and the appearance of extreme sensitivity given by the following result:

Lemma 2 If $\mathcal{A}_{0}$ contains an asymptotically stable heteroclinic attractor that is not contractible to the diagonal then $\Omega^{\text {top }}=0$.

Proof: Note that if $\mathcal{A}_{0}$ contains components that are not contractible to the diagonal then also so does $B_{\delta}\left(\mathcal{A}_{0}\right)$ for all $\delta>0$. Hence $\Omega^{\text {top }}=0$.

QED

\footnotetext{
${ }^{2}$ Another way to say this is that all pseudo-orbits in $\mathcal{A}_{0}$ have bounded phase differences.
} 


\section{Three globally coupled identical oscillators}

In case $N=3$ we write the system (2) reduced to phase differences (5) with identical oscillators (3) as

$$
\begin{aligned}
\dot{\phi}_{1}= & -\sin \left(\phi_{1}-\alpha\right)-\sin \left(\phi_{2}-\alpha\right)-\sin \left(\phi_{1}+\alpha\right) \\
& -\sin \left(\phi_{1}-\phi_{2}+\alpha\right)+r\left(2 \sin \left(2 \phi_{1}\right)+\sin \left(2 \phi_{2}\right)+\sin \left(2\left(\phi_{1}-\phi_{2}\right)\right)\right), \\
\dot{\phi}_{2}= & -\sin \left(\phi_{2}-\alpha\right)-\sin \left(\phi_{1}-\alpha\right)-\sin \left(\phi_{2}+\alpha\right) \\
& -\sin \left(\phi_{2}-\phi_{1}+\alpha\right)+r\left(2 \sin \left(2 \phi_{2}\right)+\sin \left(2 \phi_{1}\right)+\sin \left(2\left(\phi_{2}-\phi_{1}\right)\right)\right) .
\end{aligned}
$$

Observe that there are three invariant lines $\phi_{1}=0, \phi_{2}=0$ and $\phi_{1}=\phi_{2}$ that separate the torus $\mathbb{T}^{2}$ into two triangular regions: $\Phi_{1}=\left\{\left(\phi_{1}, \phi_{2}\right): \phi_{1} \in[0,2 \pi], 0 \leq \phi_{2} \leq \phi_{1}\right\}$ and $\Phi_{2}=\left\{\left(\phi_{1}, \phi_{2}\right): \phi_{1} \in[0,2 \pi], \phi_{1} \leq \phi_{2} \leq 2 \pi\right\}$ (see the phase portraits in Figures 1 and 2 ).

The phase differences for $N=3$ can be visualized as in [3] by plotting a complex phase difference

$$
\xi=\theta_{1}+e^{2 i \pi / 3} \theta_{2}+e^{4 i \pi / 3} \theta_{3}
$$

in which case permutations of the oscillators correspond to rotations and reflections in the phase space that preserve an equilateral triangle (for example, Figure 3 or [5], Figure 2). Accordingly, for system (6) we can transform the triangles $\Phi_{1}, \Phi_{2}$ into equilateral triangles by plotting in the $\xi$-plane. In the $\xi$-plane a symmetry of the system system (6) corresponds to a symmetry of an equilateral triangle.

The in-phase solution (origin) and the manifold of antiphase solutions $M^{(3)}$ are particularly significant in organizing the bifurcation behaviour of (6). Note that the latter consists of a point in each invariant triangle

$$
\left(\phi_{1}, \phi_{2}\right) \in M^{(3)}=\{(4 \pi / 3,2 \pi / 3), \quad(2 \pi / 3,4 \pi / 3)\}
$$

(we will name each of these $\mathbb{Z}_{3}$ symmetry points antiphase solutions). It can be shown that the origin and the antiphase solution have opposite stability and change them simultaneously when $\alpha$ crosses $\alpha_{0}=\arccos (2 r)$ (first the origin is an attractor and the antiphase solution is a repeller, then vice versa)

\subsection{Bifurcations for $N=3$ in the $(\alpha, r)$ plane}

The bifurcations of (6) are summarised in Figure 1 and described for a selection of slices for fixed $r$ in the section below. This analysis uses numerical path-following and simulation via the package XPPAUT/AUTO $[10,11]$.

We only plot the bifurcation diagram $\alpha \in[0, \pi]$ and $r \geq 0$, because the other cases can be inferred from the time-reversal symmetry $(x, \alpha, r) \mapsto(-x,-\alpha, r)$ and the symmetry $(x, \alpha, r) \mapsto(x+\pi, \alpha+\pi, r)$. The curve

$$
B H=\left\{(\alpha, r): r=\frac{1}{2} \cos \alpha, \alpha \in\left[0, \frac{\pi}{2}\right]\right\}
$$




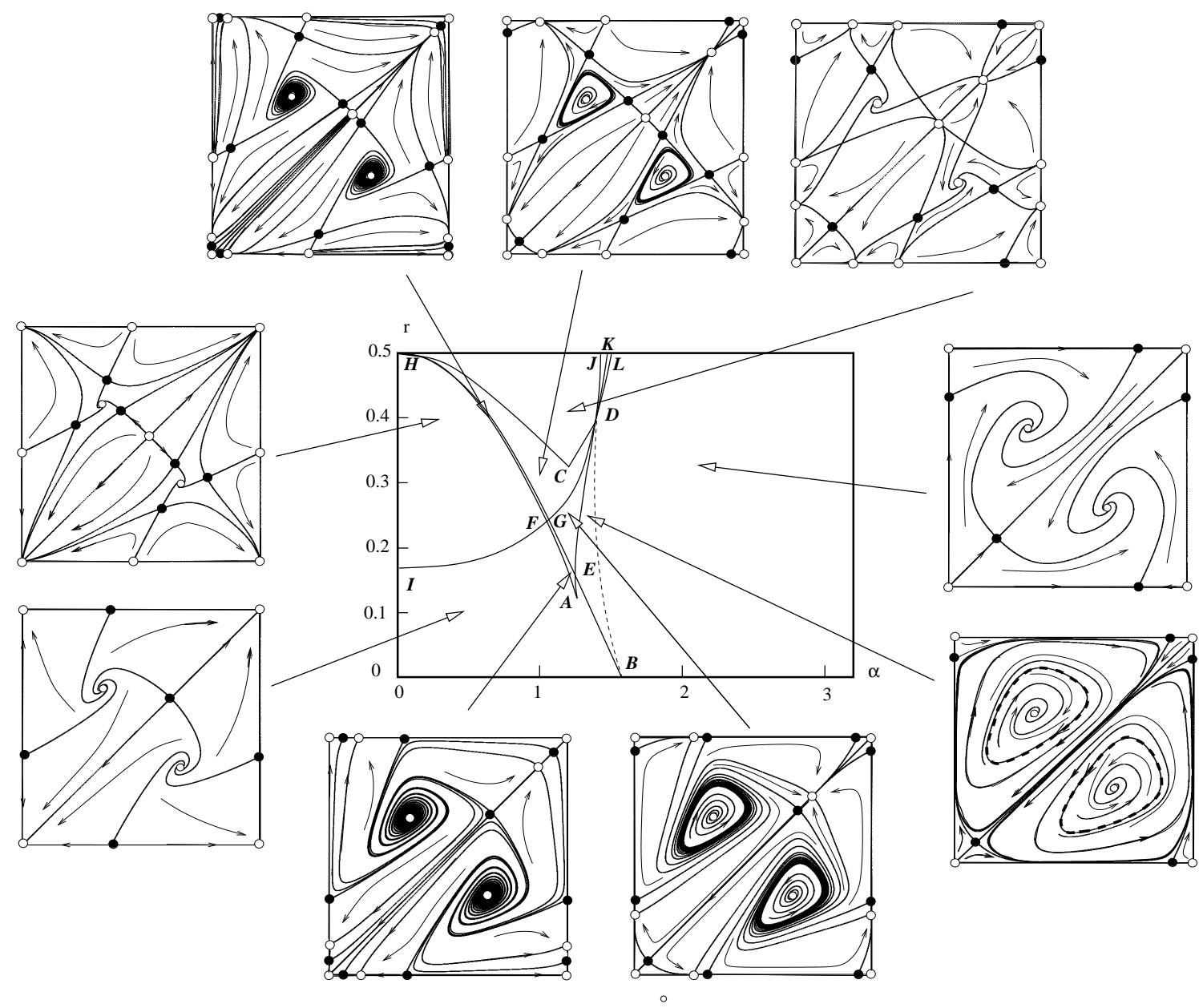

Figure 1: The parameter plane for the system (2) for $N=3$. The phase portraits surrounding the parameter plane are shown for $\left(\phi_{1}, \phi_{2}\right) \in[0,2 \pi)^{2}$. The white circles indicate sources and sinks, while the black disks indicate saddles. The codimension-one bifurcations illustrated are: $I D$ : Pitchfork bifurcation on invariant lines; $B E G H$ : Transcritical bifurcation at 0; $B E G H$ : Hopf bifurcation of antiphase solution; $H F A E D$ : Saddle-node bifurcation on invariant lines; $B E$ : $S_{3^{-}}$ transcritical homoclinic bifurcation; $E D$ : Saddle-node homoclinic bifurcation; $B D$ : Saddle-node of limit cycles; $H C D$ : $\mathbb{Z}_{3}$-heteroclinic bifurcation; $D J$ : Pitchfork bifurcation; $D K$ : Saddle-node bifurcation; $D L$ : Saddle-node bifurcation. There are codimension-two bifurcations at $A$ : cusp point; $E$ : Interaction of $S_{3}$-transcritical homoclinic and saddle-node/heteroclinic; $D$ : Interaction of saddle-node/heteroclinic and $\mathbb{Z}_{3}$-heteroclinic; $H$ : Degenerate Hopf bifurcation of antiphase solution. 


$\begin{array}{ll}I D & \text { Pitchfork bifurcation on invariant lines } \\ B E G H & \text { Transcritical bifurcation at } 0 \\ B E G H & \text { Hopf bifurcation of antiphase solution } \\ H F A E D & \text { Saddle-node bifurcation on invariant lines } \\ B E & S_{3} \text {-transcritical homoclinic bifurcation } \\ E D & \text { Saddle-node homoclinic bifurcation } \\ B D & \text { Saddle-node of limit cycles } \\ H C D & \mathbb{Z}_{3} \text {-heteroclinic bifurcation } \\ D J & \text { Pitchfork bifurcation } \\ D K & \text { Saddle-node bifurcation } \\ D L & \text { Saddle-node bifurcation }\end{array}$

Table 1: The codimension-one bifurcations for $N=3$ illustrated in Figure 1.

is the line of transcritical (in three directions) bifurcation at the origin $\left(S_{3}\right.$-transcritical bifurcation) and simultaneously it is the line of inverse supercritical Hopf bifurcation of the antiphase solutions. A transcritical bifurcation occurs when, on increasing $\alpha$, three symmetry-related saddle points (on the invariant lines $\phi_{1}=0, \phi_{2}=0$ and $\phi_{2}=\phi_{1}$ ) pass through the origin.

$S_{3}$-transcritical homoclinic bifurcation for $r \in\left(0, r_{E}\right)$. We describe the bifurcations illustrated in Figure 1 by fixing different values of the parameter $r$ and change the parameter $\alpha$ for these $r$. For $r=0$ (the Kuramoto-Sakaguchi system) we have bifurcations at the point $\alpha_{0}=\pi / 2$. There is a degenerate Hopf bifurcation at the antiphase solution, and a transcritical bifurcation at the origin that gives existence of a heteroclinic cycle between different images of 0 in the lift of $\mathbb{T}^{2}$. This heteroclinic cycle on the plane $\mathbb{R}^{2}$ splits into three homoclinic cycles when we consider the same on the torus $\mathbb{T}^{2}$.

Increasing the parameter $r$ from zero to $r_{E}$ gives a non-degenerate Hopf bifurcation (giving rise to an unstable limit cycle) and an $S_{3}$-transcritical homoclinic bifurcation as described in [4] giving rise to stable limit cycles both on the line $B E D$.

Lines $H A$ and $A D$ on the parameter plane are lines of a saddle-node bifurcation on invariant lines. We have birth (resp. disappearance) of a pair of fixed points on each of invariant lines on increasing parameter $\alpha$ through $H A$ (resp. $A D)$.

Saddle-node/heteroclinic bifurcation for $r \in\left(r_{E}, r_{D}\right)$. For $r>r_{E}$ two additional fixed points lie on each of the invariant lines after $\alpha$ has already crossed the line $E H$ of transcritical bifurcations. A heteroclinic bifurcation does not occur with the transcritical $E H$, but occurs later at the saddle-node bifurcation ED. The cycle links three pairs of saddles and and three saddle-node points lying on respective invariant lines (see also [5] for more details of this bifurcation).

If we change parameters $(\alpha, r)$ from $E$ to $D$ along the line $E D$, then the saddle point moves from $2 \pi$ to $5 \pi / 3$ and the saddle-node point will slide from $\pi / 2$ to $2 \pi / 3$ on $\phi_{1}=0$ 

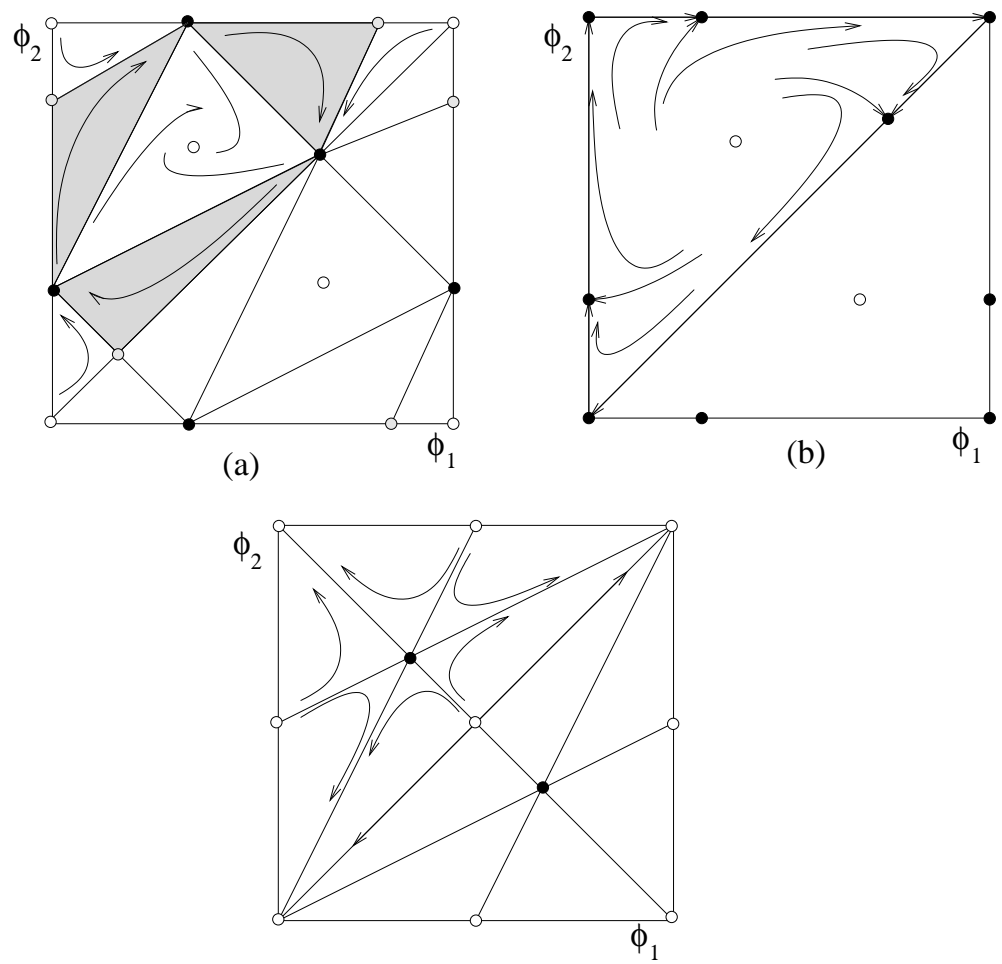

(c)

Figure 2: Phase portraits in $\phi_{i} \in[0,2 \pi)$ at the codimension two bifurcation points $D, E$ and $H$ for $N=3$; details are only shown in the upper left triangle for clarity; these are also present in the lower right triangle by the action of symmetry. The white circles indicate sources and sinks, the shaded circles indicate nonhyperbolic points while the black disks indicate saddles. (a) shows $D$; there is a cycle between saddle-nodes such that the connecting orbits (shown shaded) foliate triangles in phase space. (b) shows $E$; there are connections from the degenerately stable in-phase solution (corners) to and from saddle-node points. (c) shows $H$; there is a degenerate Hopf bifurcation at the antiphase solutions. The arrows indicate direction of flow. 
line (and symmetrically on other invariant lines). The stable limit cycle generated by the saddle-node/heteroclinic bifurcation has a roughly hexagonal shape, in contrast to such cycle generated by $S_{3}$-transcritical homoclinic bifurcation that has a roughly triangular shape. The intersection point $E$ of $S_{3}$-transcritical homoclinic and saddle-node/heteroclinic bifurcation lines has coordinates $\alpha_{E}=\arctan (3)=1.2490458, r_{E}=\sqrt{10} / 20=0.15811388$ and is illustrated in Figure 2. The mechanism of a saddle-node/heteroclinic bifurcation and its asymptotics were described in [5]. Note that the appearance of heteroclinic cycles at symmetric saddle-node and symmetric transcritical bifurcations is a general property of the system (5) for arbitrary $N \geq 3$.

Pitchfork bifurcation for $r \in\left(r_{I}, r_{D}\right)$. For $r>r_{I}=1 / 6$ there is a pitchfork bifurcation on the line $I D$ that continues up to the codimension-two bifurcation $D$ with coordinates $\alpha_{D}=5 \pi / 6-\arccos (\sqrt{21} / 14)=1.3806707$ and $r_{D}=1 / \sqrt{7}=0.37796447$. This supercritical pitchfork bifurcation is where saddles within the invariant lines bifurcate transversely to create pairs of saddles near the invariant lines. At $\alpha=0$ and on increasing $r$ through $r_{I}$ this creates a source at the mid-point of each invariant line surrounded by a pair of saddles that move towards the antiphase solutions. These saddles reach the antiphase solutions at the codimension two bifurcation $H$. The pitchfork bifurcation that occurs on the line $F D$ means that the source on the invariant line is replaced by a saddle. This saddle disappears at a saddle-node bifurcation on the invariant line on the line $D L$. The codimension-one heteroclinic cycle on the line line $E D$ generates a stable limit cycle on increasing $\alpha$.

Heteroclinic bifurcation to a stable limit cycles for $r \in\left(r_{B}, r_{D}\right)$ There is a heteroclinic cycle consisting of the three invariant lines with symmetry $S_{2} \times S_{1}$ for $(\alpha, r)$ on the curve $B E$. Also, there is a heteroclinic cycle composed of six connections, some of which lie within invariant lines, for $(\alpha, r)$ on the curve $E D$. When crossing line $B E D$ with increasing $\alpha$, a stable limit cycle is born. Therefore in the region $B E D$ we infer coexistence of a big stable and small unstable limit cycle. These disappear at a saddle-node bifurcation of limit cycles when $(\alpha, r)$ crosses $B D$; see [5] for more details.

$\mathbb{Z}_{3}$-heteroclinic bifurcation for $r \in\left(r_{C}, r_{H}=0.5\right) \quad$ The third type of symmetric heteroclinic bifurcation that takes place in this system is a $\mathbb{Z}_{3}$-heteroclinic bifurcation on the lines $C H$ and $C D$. This bifurcation occurs twice, on varying $\alpha$, for each $r \in\left(r_{C}, r_{D}\right)$ and once for $r \in\left(r_{D}, 0.5\right)$. The bifurcation occurs when there is a heteroclinic connection between pairs of three saddles related by $\mathbb{Z}_{3}$ symmetry, as shown in Figure 3. Fixing $r_{0} \in\left(r_{C}, 0.5\right)$ and changing $\alpha$ from $\alpha=\alpha_{0}=\arccos \left(2 r_{0}\right)$ we observe such a bifurcation where there are three connections meet at three saddles, destroying a stable limit cycle at the bifurcation point. On increasing the parameter $\alpha$ for $r \in\left(r_{C}, r_{D}\right)$ the $\mathbb{Z}_{3}$-heteroclinic bifurcation happens in reverse order. Following this bifurcation we obtain an unstable limit cycle.

At the $\mathbb{Z}_{3}$-heteroclinic bifurcation (on $H C D$ line) one can verify that all separatrices of saddles are straight lines given by $\phi_{2}=\left(\phi_{1}-\beta\right) / 2, \phi_{2}=2 \phi_{1}+\beta-2 \pi$ and $\phi_{2}=-\phi_{1}+\beta+2 \pi$, where $\beta$ goes from 0 to $2 \pi / 3$ as $\alpha$ goes from 0 to $\alpha_{D}$. The saddle coordinates are therefore 


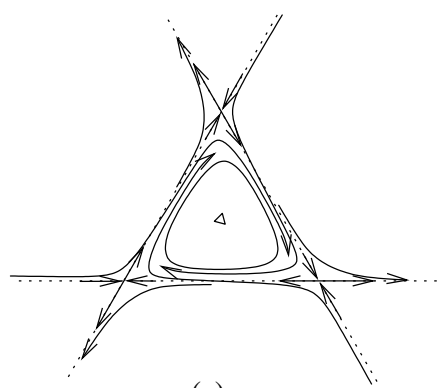

(a)

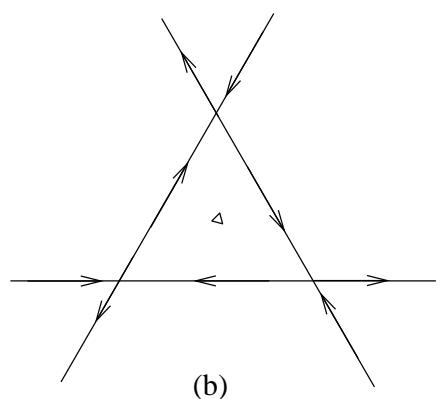

(b)

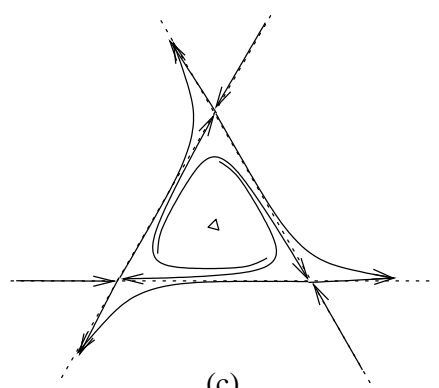

(c)

Figure 3: Schematic diagram showing detail of the $\mathbb{Z}_{3}$-heteroclinic bifurcation that occurs on the line $C D$ in parameter space for $N=3$ oscillators. Note that this occurs in the interior of the invariant triangle in a neighbourhood of the antiphase state indicated by the triangle; see text for details.

$(\beta+4 \pi / 3,2 \pi / 3),(4 \pi / 3, \beta+2 \pi / 3),(-\beta+2 \pi / 3,-\beta+4 \pi / 3)$ and similarly for other three saddles.

Interaction of saddle-node/heteroclinic and pitchfork at $D$. Seven different bifurcation lines meet at the codimension-two point $D$ shown in Figure 2. Two types of the heteroclinic bifurcation (saddle-node and $\mathbb{Z}_{3^{-}}$) take place simultaneously in such a way that they create regions in phase plane $\left(\phi_{1}, \phi_{2}\right)$ filled by trajectories of heteroclinic cycles. A large hexagonal heteroclinic cycle (generated by saddle-node bifurcation) has three points in common with the small triangular heteroclinic cycle (generated by the $\mathbb{Z}_{3}$-heteroclinic bifurcation). This results in three triangles filled with trajectories that are connections giving a set of degenerate heteroclinic cycles.

Bifurcations for $r \in\left(r_{D}, r_{H}=0.5\right)$. Lines $D J, D K$ and $D L$ are lines of pitchfork, saddlenode and saddle-node bifurcation respectively. Let us consider the region $H C D J$ for $r>r_{D}$ and increase the parameter $\alpha$. There is one sink and one source on each invariant line when $(\alpha, r) \in H C D J$ and two saddles lie off the invariant lines close to these points (created by the pitchfork when $r<r_{D}$ ). First (when $\alpha$ reaches $D J$ ) we have a pitchfork transverse to an invariant line that generates two new sinks and leaves a saddle on the invariant line. Then we have saddle-node bifurcations of these sinks as $\alpha$ crosses $D K$. The next saddlenode bifurcation of the source and saddle on the invariant line (when $\alpha$ crosses $D L$ ) gives a simple phase portrait with two sinks at the centres, the source at the origin and three saddles on the invariant lines. Note that the saddle-node points of the last bifurcation are repelling transverse to the invariant line. For this reason they are not included within any heteroclinic cycle.

Bifurcations for $r>r_{H}=0.5$ For $r>r_{H}=0.5$ we find no heteroclinic cycles or limit cycles. On increasing $r$ the phase portrait becomes topologically equivalent to the situation 
in region $J D K$ but with double the periodicity.

\subsection{Detuning and sensitivity for $N=3$}

If we allow $\Delta_{i} \neq 0$ within (5) then the bifurcation picture will change fundamentally from what is described above. However, the existence of topologically non-trivial heteroclinic cycles for identical oscillators at (a) the $S_{3}$-transcritical homoclinic bifurcation $B E$ and (b) the saddle-node heteroclinic bifurcation $E D$ implies that $\Omega^{t o p}=0$ on the line $B E D$. This suggests (see Lemma 2) that

$$
\Omega=0 \quad \text { on the line } B E D .
$$

This extends the bifurcation analysis for fixed $r$ in [5] to the two-parameter plane and explains the loss of frequency locking observed experimentally in [4] near loss of stability of the in-phase solution in a system of three coupled electronic oscillators.

\section{Four globally coupled oscillators}

\subsection{The structure of phase space for $N=4$.}

We now consider system (5) for $N=4$ posed for the phase difference variables $\left(\phi_{1}, \phi_{2}, \phi_{3}\right)$ on $\mathbb{T}^{3}$ and again with $\Delta_{i} \equiv 0$. As already mentioned, all planes $\phi_{i}=0, i=1,2,3$, are invariant and all lines $\phi_{i}=0, \phi_{j}=0, i, j=1, \cdots, 3, \phi_{1}=\phi_{2}=\phi_{3}$ are invariant as well. In the terms of symmetry groups, these lines have $S_{3} \times S_{1}$ isotropy $[3,9]$. Also the planes $\phi_{i}=\phi_{j}$, $i=1,2,3$, are invariant. Thus the diagonals of the cube faces $\phi_{i}=\phi_{j}, \phi_{k}=0, i \neq j \neq k$, $i, j, k=1,2,3$, are invariant. These lines have isotropy $S_{2} \times S_{2}$.

If we take the cube $[0,2 \pi)^{3}$ modulo its main diagonal we can divide it into six equal volume tetrahedra with the help of the above described invariant planes. Each tetrahedron is an invariant region corresponding to points on $\mathbb{T}^{4}$ that lift to the set

$$
\left\{\left(\theta_{1}, \theta_{2}, \theta_{3}, \theta_{4}\right): \theta_{\sigma(1)} \leq \theta_{\sigma(2)} \leq \theta_{\sigma(3)} \leq \theta_{\sigma(4)} \leq \theta_{\sigma(1)}+2 \pi\right\} .
$$

for some permutation $\sigma \in S_{4}$. For the particular case where $\sigma$ is the identity this invariant set is called the canonical invariant region [3]. These tetrahedra have faces with $S_{2}$ isotropy. Of the edges, four have $S_{3} \times S_{1}$ isotropy and two have $S_{2} \times S_{2}$ isotropy. The manifold of antiphase solutions $M^{(4)}$ consists of six direct lines that connect the centres of the cube faces. Each of these lines belongs to one of tetrahedra and has $\mathbb{Z}_{2}$ isotropy. The centre of manifold $M^{(4)}$ is an antiphase solution with $\mathbb{Z}_{4}$ isotropy and a point of intersection of lines with $S_{2} \times S_{2}$ and $\mathbb{Z}_{2}$ isotropy has $\left(S_{2}\right)^{2} \times{ }_{s} \mathbb{Z}_{2}$ isotropy where $\times_{s}$ indicates a semidirect product. Hence we split the torus of phase differences into six solid tetrahedra of the form shown in Figure 4.

Let us imagine that four points of cube $(0,0,0),(2 \pi, 0,0),(2 \pi, 2 \pi, 0),(2 \pi, 2 \pi, 2 \pi)$ are connected in the sequentially closed curve $\gamma_{1}$ and this curve has some direction. In the same way we can define similar curves $\gamma_{i}, i=2, \cdots, 6$ for other tetrahedra. From this point we consider only the dynamics within one tetrahedron as the dynamics on all others is given by symmetry. 


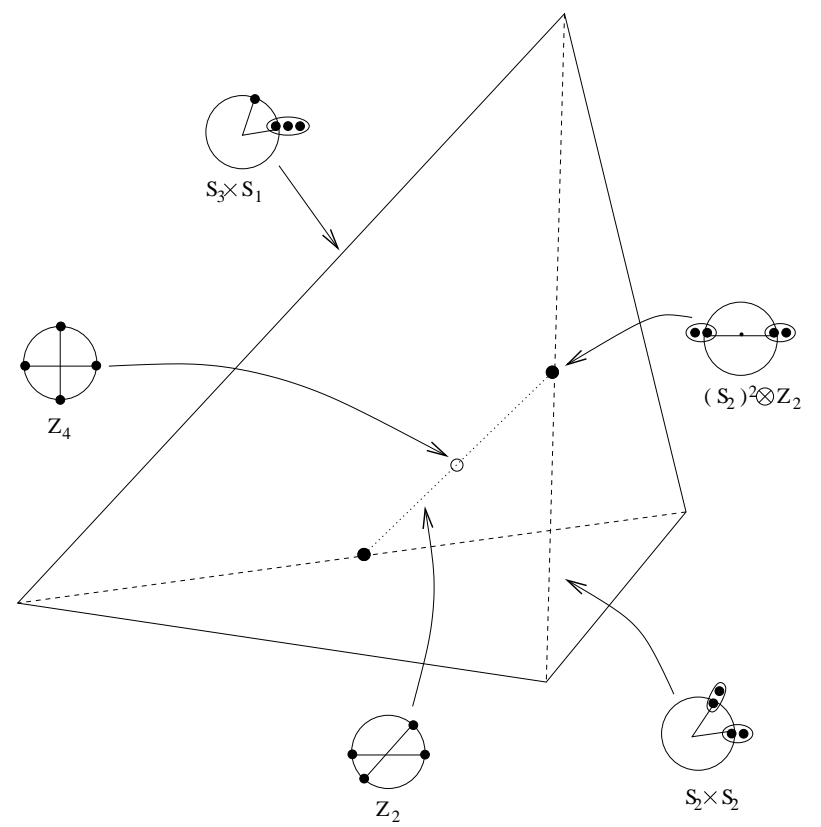

Figure 4: Diagram showing the phase space in terms of one invariant tetrahedron for the case of identical phase oscillators with $N=4$. This shows the relationship between the subspaces with differing symmetries. The point at the centre is the antiphase solution with $\mathbb{Z}_{4}$ symmetry; the points on the faces of the invariant tetrahedron have $S_{2}$ symmetry. 


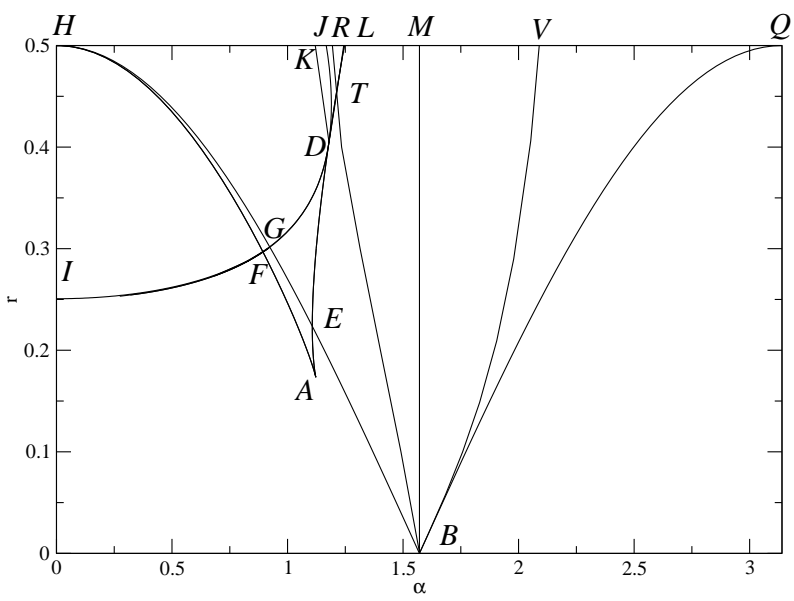

Figure 5: Bifurcation diagram for $N=4$ oscillators in the $(\alpha, r)$. See text and Table 2 for a description of the bifurcation lines; there are robust heteroclinic cycles between two cluster states in the region outlined by $B E D T L V$ that are attractors to the left of the line $B M$. There is a complicated sequence of bifurcations near the point $D$ that is not shown in detail in this diagram.

\subsection{Bifurcation structure for $N=4$.}

A diagram showing the main bifurcation structure for $N=4$ is given in Figure 5 with the codimension-one bifurcations listed in Table 2. Firstly we consider the case $r=0$ (the Kuramoto-Sakaguchi system). For $\alpha=0$ we have one attractor that is origin and one repeller that is the manifold of fixed points $M^{(4)}$. Also there are saddles with all coordinates 0 or $\pi$. For increasing parameter $\alpha$ all saddles begin to move along the invariant lines in the (or reverse) direction of $\gamma_{i}$. When $\alpha=\pi / 2$, the transcritical bifurcation (along $S_{3} \times S_{1}$ invariant lines) at the origin and the Hopf bifurcation on the $M^{(4)}$ take place. At this moment each tetrahedron is filled with closed trajectories and all invariant planes are filled with parts of heteroclinic cycles. Each connection $\gamma(\phi)=W^{u}\left(P_{1}(\phi)\right) \bigcap W^{s}\left(P_{2}(2 \pi-\phi)\right)$ links the two degenerate saddles $P_{1}(\phi), P_{2}(2 \pi-\phi), \phi \in[0, \pi]$, where $\phi$ parameterises these lines (we have three pairs of $P_{1}, P_{2}$ since there are three $S_{2} \times S_{2}$ lines on the torus $\mathbb{T}^{3}$ ). We obtain continuous sets of heteroclinic cycles because the whole $S_{2} \times S_{2}$ invariant lines are filled with saddle points as in Figure 6. The origin and manifold $M^{(4)}$ change their stability at the moment of this bifurcation and heteroclinic cycles disappear except at $\alpha=\pi / 2$.

\subsection{Heteroclinic cycles for $N=4$.}

For $r \in(0,0.5)$, Figure 5 shows that there are two curves $\alpha_{1}(r)<\alpha_{2}(r)$ such that heteroclinic cycles exist when $\alpha \in\left(\alpha_{1}(r), \alpha_{2}(r)\right)$. Despite the various types of bifurcation creating them, all heteroclinic cycles in this case have common properties:

1. All heteroclinic cycles consist of a union of $\Gamma_{1}$ and $\Gamma_{2}$, subsets of two different $S_{2}$ 


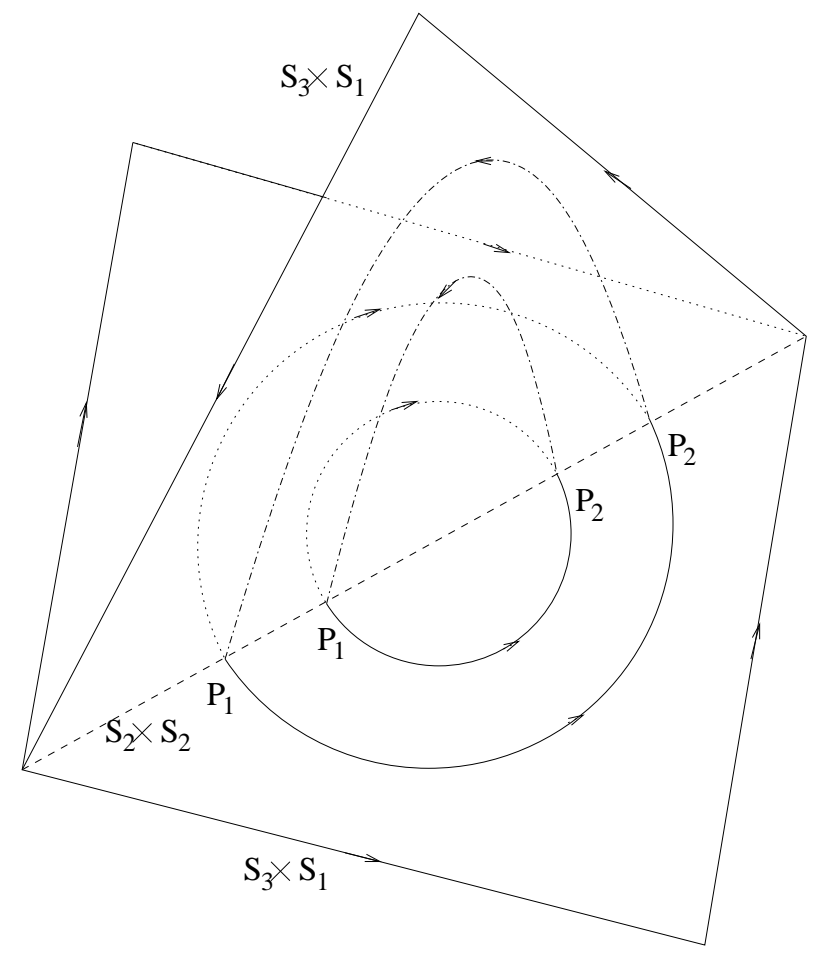

Figure 6: Continua of heteroclinic cycles for $N=4$ for $(\alpha, r)=(\pi / 2,0)$ (point $B$ in Figure 5). For this degenerate situation there is a manifold of fixed points on the $S_{2} \times S_{2}$ invariant subspace. 
$B E G H \quad$ Transcritical-pitchfork bifurcation at 0

$B Q \quad$ Inverse pitchfork bifurcation of saddles with $\left(S_{2}\right)^{2} x_{s} Z_{2}$ symmetry

$B V \quad$ Pitchfork/heteroclinic bifurcation of solutions with symmetry $S_{2} \times S_{2}$ (transverse to $S_{2} \times S_{2}$ direction)

$B M \quad$ Hopf bifurcation of antiphase solutions $\left(\mathbb{Z}_{4}\right)$ and change of stability of robust heteroclinic cycles

$H A E D \quad$ Saddle-node bifurcation to solutions with symmetry $S_{3} \times S_{1}$

IFGD Transcritical bifurcation of solutions with symmetry $S_{3} \times S_{1}$ at the source $U_{i}$

$D K \quad$ Saddle connection bifurcation (not heteroclinic) in subspace with symmetry $S_{2}$

DJ Transcritical bifurcation of solutions with symmetry $S_{3} \times S_{1}$ at the $\operatorname{sink} W_{i}$

DTL Saddle-node bifurcation inside tetrahedra on $S_{2}$ plane

BTR Pitchfork bifurcation of limit cycles within tetrahedron

$I F G D^{\prime} \quad$ A saddle-node bifurcation line lies to the right of the line $I F G D$ and very close to it

$D J^{\prime} \quad$ A saddle-node bifurcation line lies between $D K$ and $D J$ (the bifurcation occurs on $W_{i} V_{i}$ in phase space)

$D L^{\prime}, D L^{\prime \prime} \quad$ Two saddle-node bifurcation lines are between $D J$ and $D L$, the second of these bifurcations happens within the $S_{3} \times S_{1}$ invariant line.

Table 2: A list of the codimension-one bifurcations for $N=4$ including those illustrated in Figure 5 .

invariant planes connected by $S_{2} \times S_{2}$ invariant line. Each of these two lines $\Gamma_{1}$ and $\Gamma_{2}$ consists of several parts:

$$
\Gamma_{i}=\bigcup_{j=1}^{N} \Gamma_{i j}, \quad i=1,2, \quad j=1, \cdots, m
$$

where $m$ can change from 1 to 5 depending on the heteroclinic cycle type.

2. $\Gamma_{1}$ and $\Gamma_{2}$ connect two saddles $P_{1}, P_{2}$, so that $W^{u}\left(P_{1}\right)=\Gamma_{11}, W^{s}\left(P_{2}\right)=\Gamma_{1 N}, W^{u}\left(P_{2}\right)=$ $\Gamma_{21}, W^{s}\left(P_{1}\right)=\Gamma_{2 N}$ and other two pairs of stable manifold of these saddles belong to invariant $S_{2} \times S_{2}$ line.

For any fixed $r \in(0,0.5)$ and increasing $\alpha$ saddles $P_{1}$ and $P_{2}$ appear as a result of the subcritical pitchfork bifurcation that occurs at the origin when $\alpha_{-}=\arccos \left(2 r_{0}\right)$ and disappear as a result of an subcritical pitchfork bifurcation at the middle of $S_{2} \times S_{2}$ invariant lines (a point with $\left(S_{2}\right)^{2} \times_{s} \mathbb{Z}_{2}$ symmetry) when $\alpha_{+}=\pi-\arccos \left(2 r_{0}\right)$. The lines indicating these pitchfork bifurcations $(\alpha, r)$ are $B H$ and $B Q$ respectively. The saddles $P_{1}$ and $P_{2}$ on invariant $S_{2} \times S_{2}$ line are $\phi\left(P_{1}\right)=\arccos \left(\frac{\cos \alpha}{2 r}\right), \phi\left(P_{2}\right)=2 \pi-\arccos \left(\frac{\cos \alpha}{2 r}\right)$ respectively. The system (5) has the same eigenvalues at points $P_{1}$ and $P_{2}$ which are expressed by the formulae:

$$
\begin{gathered}
\lambda_{1}(\alpha, r)=-\frac{2}{r}\left(4 r^{2}-\cos ^{2} \alpha\right), \\
\lambda_{2,3}(\alpha, r)=-\frac{1}{r}\left(\cos \alpha(2 r-\cos \alpha) \mp \sin \alpha \sqrt{4 r^{2}-\cos ^{2} \alpha}\right) .
\end{gathered}
$$


We note that the existence of saddle points $P_{1}$ and $P_{2}$ is a necessary but not sufficient condition of heteroclinic cycles existence, i.e. $\left[\alpha_{1}, \alpha_{2}\right] \subset\left[\alpha_{-}, \alpha_{+}\right]$, and there are two reasons for this. The first reason is that the invariant manifolds of saddles $P_{i}, i=1,2$, changes stability such that $\operatorname{dim} W^{u}\left(P_{i}\right)=1, \operatorname{dim} W^{s}\left(P_{i}\right)=2$ before the bifurcation at $\alpha=\alpha_{2}$ and $\operatorname{dim} W^{u}\left(P_{i}\right)=2, \operatorname{dim} W^{s}\left(P_{i}\right)=1$ after this bifurcation. The second reason is that the chain of invariant manifolds $\Gamma_{i j}, j=1, \cdots, m$, (parts of the heteroclinic cycle that belong to the invariant planes) can be broken on the $S_{3} \times S_{1}$ invariant lines or close to them by bifurcations of various types.

\subsection{Bifurcation to heteroclinic cycles for $N=4$}

The first type of heteroclinic bifurcation occurs for $r_{0} \in(0, \sqrt{5} / 10)$ and is an $S_{4}$-bifurcation of transcritical-pitchfork type. We have a transcritical bifurcation at the origin that takes place along four $S_{3} \times S_{1}$ invariant lines and simultaneously we have a pitchfork bifurcation at this point along three $S_{2} \times S_{2}$ invariant lines. These two bifurcations occur when $\alpha=$ $\alpha_{-}=\arccos \left(2 r_{0}\right)$, i.e. on the $B H$-line in a two-parameter plane (the same line $B H$ of the transcritical bifurcation was for $N=3$ ). The transcritical bifurcation changes stability of the origin. The pitchfork bifurcation generates pair saddle points $P_{1}, P_{2}$ mentioned above. Thus there are heteroclinic cycles $\gamma_{i}, i=1, \cdots, 6$, (in the 3 -dimensional cube), each of them on the edges of its tetrahedron, i.e. each of them consists of four invariant $S_{3} \times S_{1}$ lines as in Figure 7.

On the other hand, we have four homoclinic orbits on the torus $\mathbb{T}^{3}$ that connect from and to the origin. In terms of $\Gamma$ we have $\Gamma_{i}=\Gamma_{i 1} \cup \Gamma_{i 2}, i=1,2$, and all $\Gamma_{i j}$ are invariant $S_{3} \times S_{1}$ lines. On increasing $\alpha>\alpha_{-}$the saddles $P_{1}$ and $P_{2}$ move along $S_{2} \times S_{2}$ invariant lines and pull $\Gamma_{i}$ into the tetrahedra. After bifurcation $\Gamma_{i}$ leaves the origin with two saddles in $S_{2} \times S_{2}$ direction and loses the fixed point in the orthogonal direction. Thus each $\Gamma_{i}$ consists now only of one part and each heteroclinic cycle consists of two parts; see Figure 8. This means we obtain two heteroclinic cycles in each tetrahedron.

We have described the appearance of heteroclinic cycles with the first type of bifurcation that happens for $r \leq \sqrt{5} / 10$ and $\alpha_{1}$ on the line $B H$. For other values of $r$ and increasing $\alpha$ we have different bifurcation types that generate heteroclinic cycles. Regardless of the types of the bifurcation appearance heteroclinic cycles exist on some interval $\alpha \in\left(\alpha_{1}, \alpha_{2}\right)$ and are destroyed in the same way for any $r \in(0,0.5)$. Before the disappearance of bifurcation any heteroclinic cycle consists of two curves $\Gamma_{11}, \Gamma_{12}$ connecting $P_{1}$ and $P_{2}$ (Figure 8). Saddle points $P_{1}, P_{2}$ disappear with the pitchfork bifurcation on the line

$$
B Q=\left\{(\alpha, r): r=-\frac{1}{2} \cos \alpha, \alpha \in(\pi / 2, \pi)\right\}
$$

when $\alpha=\alpha_{+}$but heteroclinic cycles disappear earlier with another pitchfork bifurcation on the line

$$
B V=\left\{(\alpha, r): r=\frac{\cos \alpha}{2 \cos (2 \alpha)}, \alpha \in(\pi / 2,2 \pi / 3)\right\}
$$




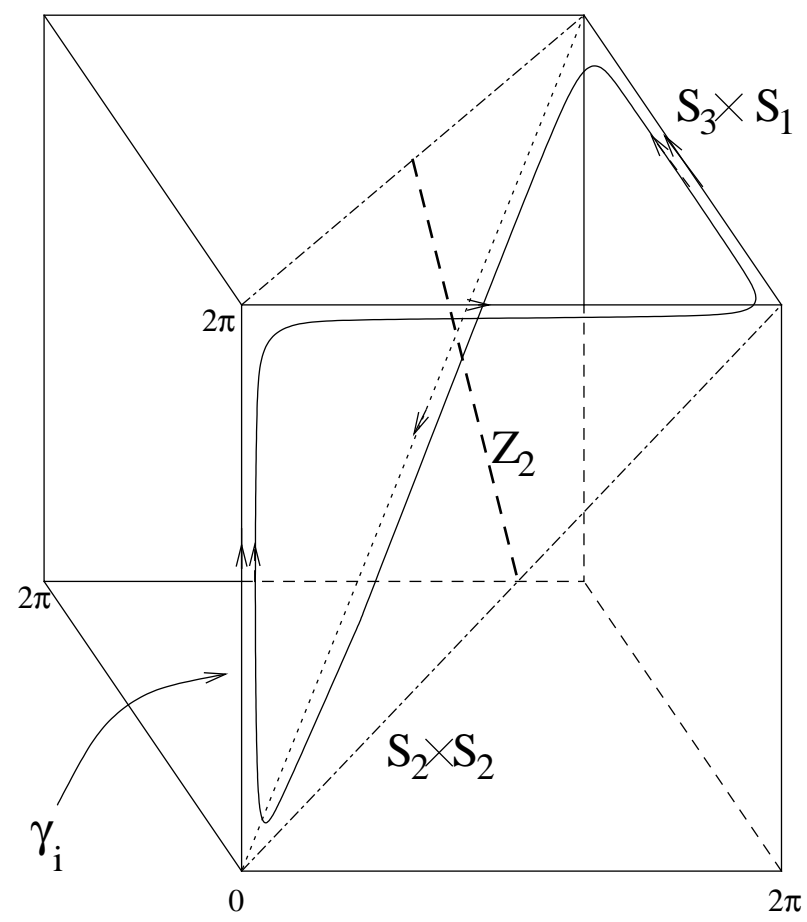

Figure 7: Schematic diagram showing one of the heteroclinic cycles for $N=4$ in the $\phi_{i}$ coordinates just to the right of the transcritical-pitchfork heteroclinic bifurcation line ( $B E$ in Figure 5$)$. Note that there is a saddle-type periodic orbit near the cycle. 


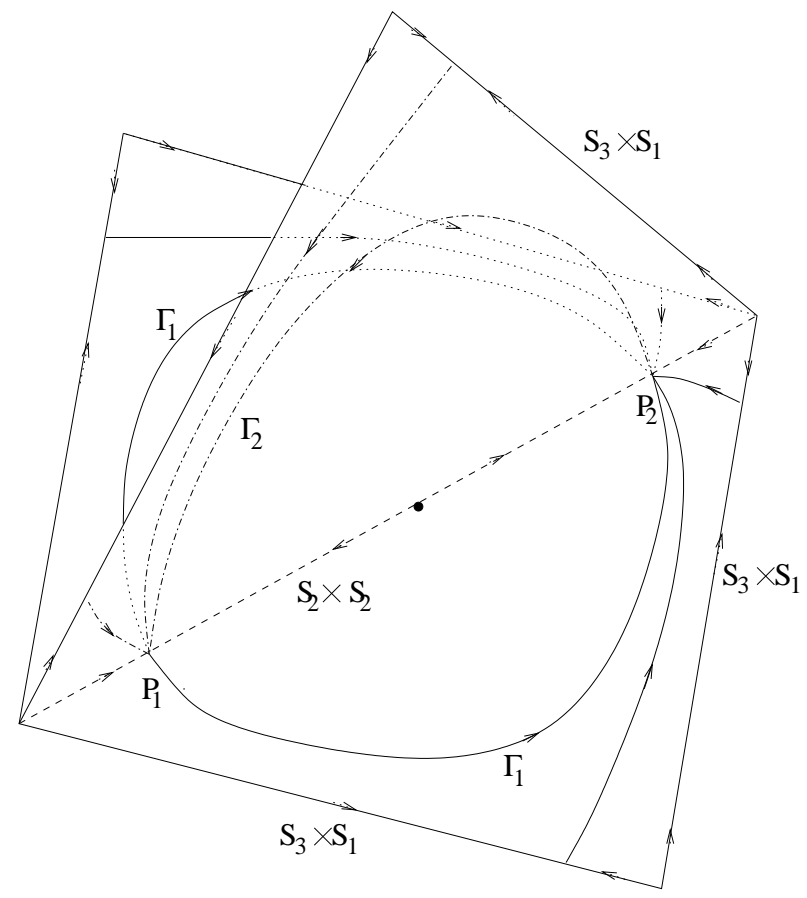

Figure 8: Schematic showing robust heteroclinic cycles inside area $B E D T L V$ on Figure 5 for $N=4$ that are attracting on the left and repelling on the right side of $B M$. We investigate a range of bifurcations leading to the creation of such cycles. 
when $\alpha=\alpha_{2}$. This last supercritical pitchfork bifurcation occurs in another transverse direction to the $S_{2} \times S_{2}$ invariant line directions at points $P_{2}$ and $P_{1}$. Thus two new saddles appear on the invariant plane where $\Gamma_{1}=W^{u}\left(P_{1}\right)$ lies and two other ones appear in the plane where $\Gamma_{2}=W^{u}\left(P_{2}\right)$ lies. Eigenvalues $\lambda_{2}(\alpha, r)$ of points $P_{1}, P_{2}$ change their signs from negative to positive on the curve $B V$. These points become unstable in both transverse directions to the invariant line, and the heteroclinic cycles split. This is another route for appearance of heteroclinic cycles. We note that $B Q$ and $B V$ are bifurcation lines within $\alpha \in(\pi / 2, \pi)$ and $r \in(0,0.5)$. Thus heteroclinic cycles exist at least for any $\alpha \in\left(\pi / 2, \alpha_{2}\right)$, and disappear (appear) at the pitchfork/heteroclinic bifurcation on $B V$.

\subsection{Stabilities for $N=4$}

The eigenvalues $\lambda_{1}(\alpha, r)$ of saddles $P_{1}, P_{2}$ in the $S_{2} \times S_{2}$ direction are negative for any parameters $(\alpha, r)$ in the area $B Q H$ of Figure 5 (i.e. when $\alpha \in\left(\alpha_{-}, \alpha_{+}\right)$and $\left.r \in[0,0.5]\right)$. Hence these saddles attract along the $S_{2} \times S_{2}$ invariant lines. The sum of other pairs of eigenvalues $\sigma(\alpha, r)=\lambda_{2}(\alpha, r)+\lambda_{3}(\alpha, r)$ is negative for $\alpha \in\left(\alpha_{-}, \pi / 2\right)$ and is positive for $\alpha \in\left(\pi / 2, \alpha_{+}\right)$.

Stabilities of heteroclinic cycles From the above we can conclude that heteroclinic cycles $\Gamma_{1} \bigcup \Gamma_{2}$ attract when they exist and $\alpha<\pi / 2$ and they repel when $\alpha>\pi / 2$. The straight line

$$
B M=\{(\alpha, r): \alpha=\pi / 2\}
$$

is the line of stability change (resonance) of heteroclinic cycles, which for this system cooincides with the line of Hopf bifurcations of the antiphase solutions with $\mathbb{Z}_{4}$ symmetry. A subcritical bifurcation from the heteroclinic cycles occurs transverse to the $S_{2}$ invariant planes when $\alpha$ intersects line $B M$. The unstable heteroclinic cycle becomes stable and in doing so generates two saddle limit cycles when $\alpha$ decreases giving unstable limit cycles as illustrated in FIgure 7 for $\alpha$ slightly less than $\pi / 2$. .

Stability of the antisynchronized set We consider the antisynchronized set $M^{(4)}$ in the interior of a tetrahedron (line with $\mathbb{Z}_{2}$ symmetry, Figure 4). The centre of $M^{(4)}$ (with $\mathbb{Z}_{4}$ symmetry) is attractor along the manifold for any $\alpha, r$ in the region considered. It is a saddle-focus for $\alpha \in[0, \pi / 2))$ and a sink for $\alpha \in(\pi / 2, \pi)$. The centre of invariant manifold $M^{(4)}$ changes its stability as a result of the supercritical Hopf bifurcation on $B M$.

Summarising, if $\alpha$ is slightly less than $\pi / 2$ we have two stable heteroclinic cycles on the tetrahedron faces, a stable limit cycle inside the tetrahedron and two saddle limit cycles that separate them. On decreasing $\alpha$ to cross line BTR we obtain a subcritical pitchfork bifurcation of the stable limit cycle and two saddle limit cycles. For smaller $\alpha$ the saddle limit cycle is the only limit cycle inside the tetrahedron. In the case $r \in[0, \sqrt{5} / 10]$ this saddle limit cycle appears together with heteroclinic cycles in a transcritical-pitchfork bifurcation. At this bifurcation an attracting heteroclinic cycle (that consists of four parts) is created that bifurcates into two stable heteroclinic cycles and a saddle limit cycle, on increasing $\alpha$ 


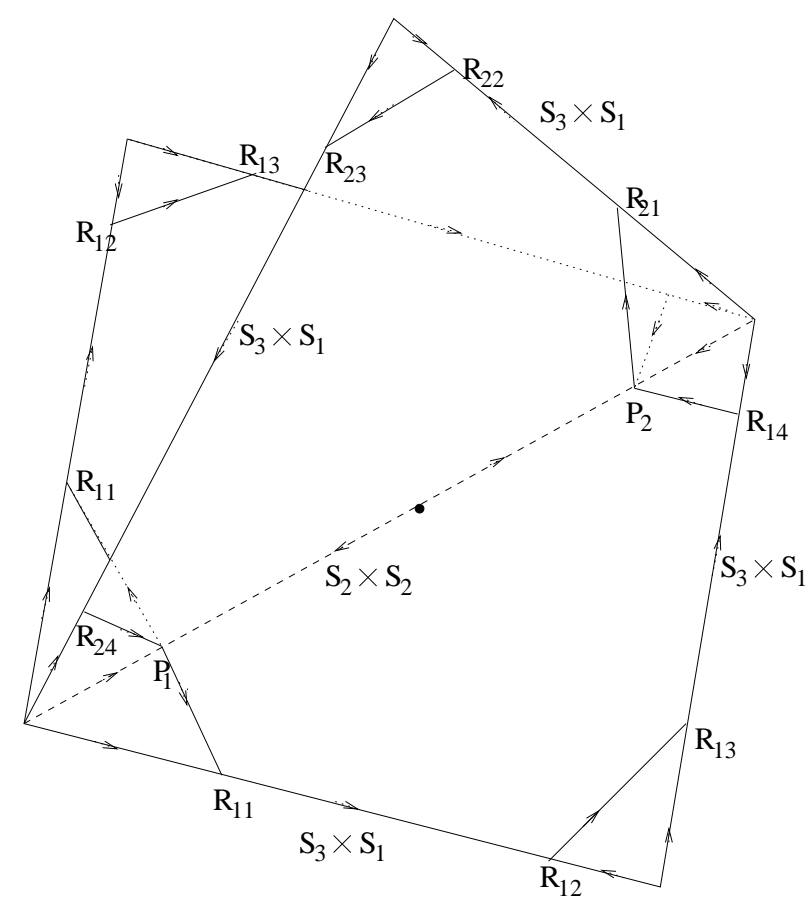

Figure 9: Bifurcation to heteroclinic cycles for $N=4$ on the line $E D$ in Figure 5 . There are two dimensional sets of connections between the saddle-nodes $R_{11} \rightarrow R_{13}$ and $R_{13} \rightarrow R_{14}$.

through $B H$. We note that then the heteroclinic cycles coexist with just the saddle limit cycle, these heteroclinic cycles are the only attractors in our system. As in the case $N=3$, the line of transcritical homoclinic bifurcations to the origin connects to a line of saddle-node bifurcations. They meet at $E$ where $\left(\alpha_{E}, r_{E}\right)=(\arctan (3), \sqrt{10} / 20)$ in Figure 5.

The saddle-node/heteroclinic bifurcation For $N=4$ the saddle-node bifurcation that happens on invariant lines ( $H A$ in the $(\alpha, r)$-plane) can break up the heteroclinic cycle. For $r>r_{E}$ this scenario is not possible since the unstable manifold $W^{u}\left(P_{1}\right)$ limits to a sink. The same is with $W^{s}\left(P_{2}\right)$ and the closest source. The saddle connection bifurcation that happens on the invariant $S_{3} \times S_{1}$ lines and invariant planes close to these lines causes the appearance of a heteroclinic cycle. The simplest scenario of this bifurcation is for $r \in\left(r_{E}, r_{D}\right)$ as in Figure 9. In this case each of $\Gamma_{i}, i=1,2$, consists of 5 parts. If we consider the tetrahedron on the whole then we can see that two heteroclinic cycles of the tetrahedron have four common parts $\left(R_{11} R_{12}, R_{13} R_{14}, R_{21} R_{22}, R_{23} R_{24}\right)$. It can be shown that there exist four quasi-triangular 2-dimensional areas (like $R_{24} P_{1} R_{11}$ ) filled with trajectories going in the same direction as in Figure 10. Thus we have 2-dimensional sets of heteroclinic cycles inside each tetrahedron that consist of a union of 4 triangular regions and 4 connecting lines. One of heteroclinic cycles of this set has 12 parts: $P_{1} R_{11} R_{12} P_{1}^{\prime} R_{13} R_{14} P_{2} R_{21} R_{22} P_{2}^{\prime} R_{23} R_{24}$. With increasing $\alpha$ we obtain the usual heteroclinic cycles for $N=4$ that change their stability when $\alpha=\pi / 2$ and disappear when $\alpha$ crosses $B V$. 


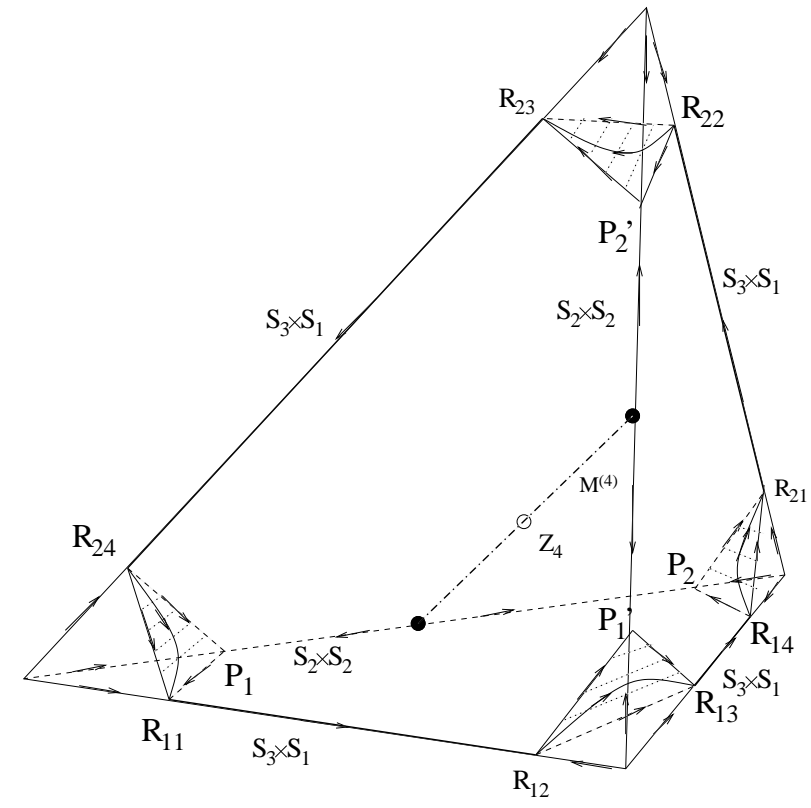

Figure 10: Detail of the interior connections that exist in the case shown in Figure 9 for $N=4$. 


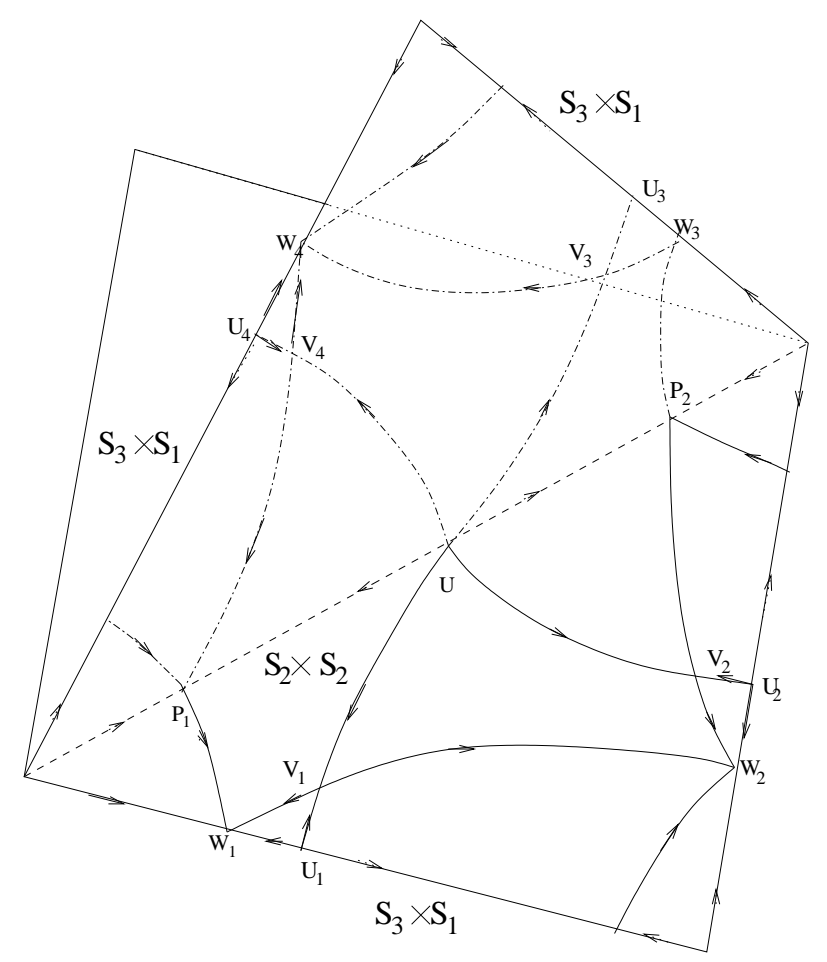

Figure 11: Global connections for parameters belonging to $H G D K$ in Figure 5 and $N=4$.

Figure 11 schematically shows the phase portrait on the invariant planes when parameters belong to the area $H G D K$. We now demonstrate that the scenario of heteroclinic cycle appearance must be more complicated than just saddle-node bifurcation of two points on invariant $S_{3} \times S_{1}$ lines. Line $I D$ of Figure 5 represents transcritical and saddle-node bifurcations involving a source that lies in the $S_{3} \times S_{1}$ invariant line and six saddles which belong to invariant planes. The two lines of bifurcations lie very close to each other. The bifurcation sequence happens on invariant planes and is schematically represented by Figure 12 a)-e). Point $I$ has coordinates $\alpha=0$ and $r=1 / 4$. There are twelve saddles around each source for any $r>1 / 4$ and $\alpha=0$. Pitchfork bifurcations of nine saddles on increasing $\alpha$ reduce these twelve saddles to six saddles that lie on invariant planes. Line $I D$ crosses the saddle-node bifurcation line $H A$ at $G$ and the transcritical bifurcation line $B H$ at $F$. Therefore we have three main possibilities to obtain a heteroclinic cycle for $r>r_{G}=0.303$ approximately.

1. On increasing $\alpha$, unstable point $U_{i}$ participates in some bifurcations with point $V_{i}$ and becomes a saddle point. This saddle connects with stable point $W_{i}$ at a saddlenode/heteroclinic bifurcation, as shown in Figure 12. This sequence bifurcations occurs for $r \in(0.302,0.404)$ approximately.

2. On increasing $\alpha$, the stable point $W_{i}$ bifurcates to three stable points inside the tetrahedron on three invariant planes and transforms into the saddle before it disappears in a saddle-node bifurcation with point $U_{i}$. The stable points created participate in 


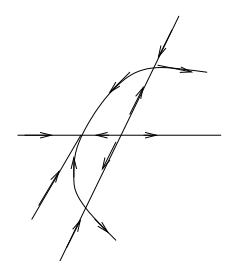

a)

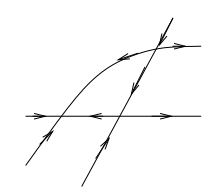

d)

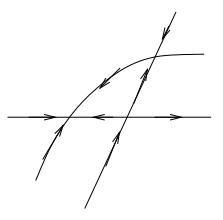

b)

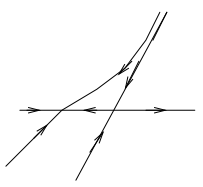

e)

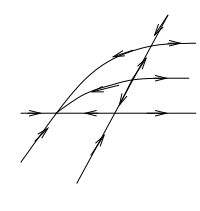

c)

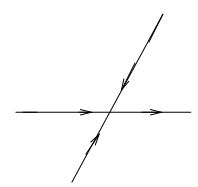

f)

Figure 12: The sequence of bifurcations on increasing $\alpha$ for the connections shown in Figure 11 and $r \in(0.302,0.404)$. The situation in f) corresponds to crossing the line $D L$ in Figure 5.

a saddle-node bifurcation with points $V_{i}$ on increasing $\alpha$ as shown in Figure 13(a). Line $D J$ on the bifurcation diagram represents the first saddle-node bifurcation Figure $13(\mathrm{~b})$. We note that the latter saddle-node bifurcation occurs inside the tetrahedron in the invariant plane, not on the invariant $S_{3} \times S_{1}$ lines. We note that the saddle connection bifurcation $K D$, giving reorganization of separatrices $V_{1} W_{2}$ and $U V_{2}$ takes place before this bifurcation occurs. After the saddle connection bifurcation we have the phase portrait shown in Figure 14. We note also that the sequence of bifurcations (shown in Figure 13) is different with the g)-h) bifurcation happening before e)-f) with increasing $\alpha$ and values of $r$ close to 0.5 .

3. When parameter $r$ is in a small interval near $r=0.404$ the sequence of bifurcations is rather more complicated. In the previous two cases, one of nodes $U_{i}$ or $W_{i}$ became saddle (unstable or stable in transversal to invariant lines direction respectively) after some bifurcations and then this saddle interacts with other nodes, disappearing in saddle-node bifurcation. In this case there is a direct interaction of these nodes as shown in Figure 15 as the parameter $\alpha$ changes on a very small interval. Further we find the heteroclinic bifurcation illustrated in Figures 16. The sequence of bifurcations shown in Figures 15 ends with (e) shown in Figure 16(a) just before the heteroclinic bifurcation. At bifurcation, a heteroclinic cycle consists of four parts which do not have common points with $S_{3} \times S_{1}$ invariant lines Figure 16(b). After bifurcation, saddle points $R_{i 1}$ coexist with the heteroclinic cycle for small increase of $\alpha$ and then disappear at a saddlenode bifurcation. Heteroclinic bifurcations also occur for small $r \in(0.404,0.04045)$ as a continuation ofthe bifurcation sequence shown on Figure 15 a) -d) with further saddlenode bifurcations on the invariant line. Thus each heteroclinic cycle consists of four parts which do not have common points with $S_{3} \times S_{1}$ invariant lines. After bifurcation saddle points $R_{i 1}$ coexist with the heteroclinic cycle for small increase of $\alpha$ and then 


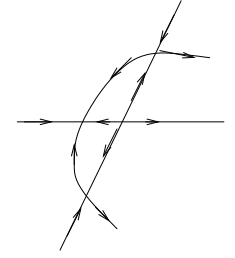

a)

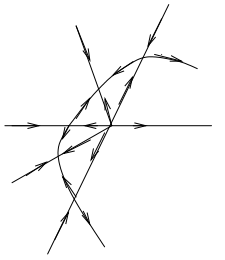

d)

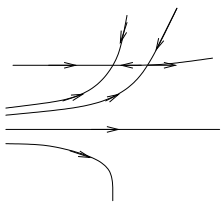

g)

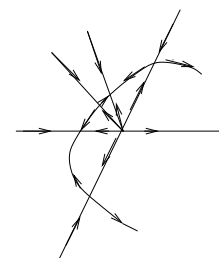

b)

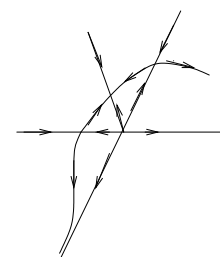

e)

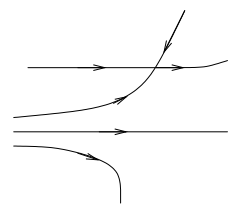

h)

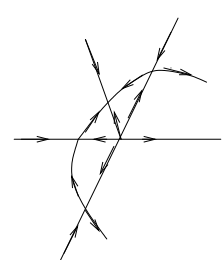

c)

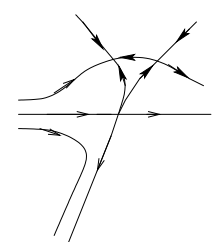

f)

Figure 13: The sequence of bifurcations on increasing $\alpha$ for the connections shown in Figure 11 and $r>0.4045$. The situation in $\mathrm{f}$ ) corresponds to crossing the line $D L$ in Figure 5 . 


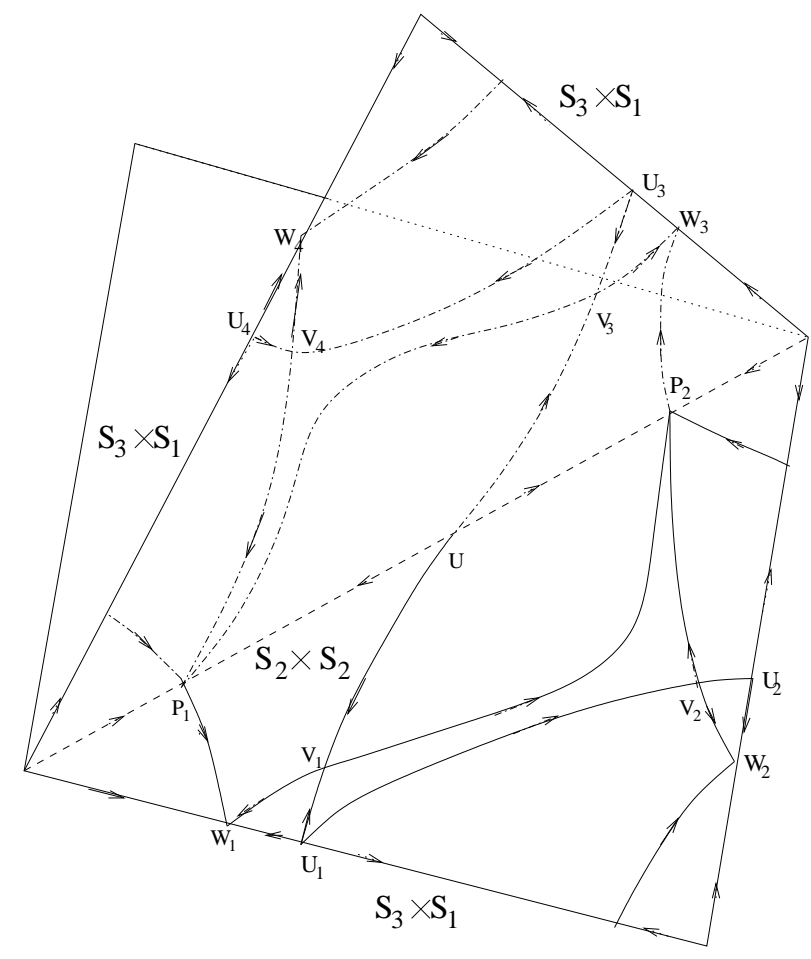

Figure 14: Global connections for parameters belonging to KDJ and for $N=4$.

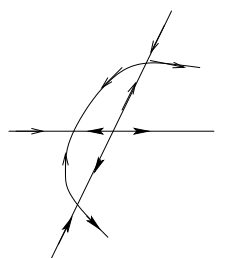

a)

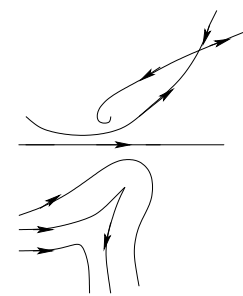

d)

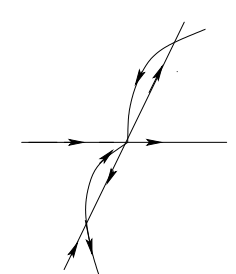

b)

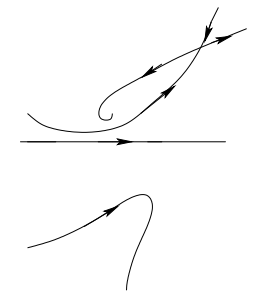

e)

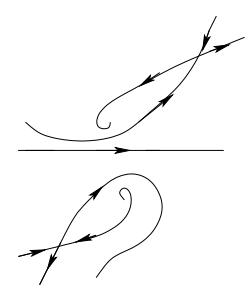

c)

Figure 15: The sequence of bifurcations on increasing $\alpha$ for the connections shown in Figure 11 and $r$ close to 0.404 . The situation in b) corresponds to crossing the line $D L$ in Figure 5 . 
disappear in the saddle-node with the saddle-node bifurcation.

\subsection{Summary of heteroclinic bifurcations for $N=4$}

We find five distinct types of codimension-one bifurcation to robust heteroclinic cycles in the $(\alpha, r)$ plane; the curves correspond to their labelling in Figure 5.

1. TCPFH Transcritical-pitchfork heteroclinic at the origin on the line $B E$

2. SNH Saddle-node at invariant $S_{3} \times S_{1}$ lines on the line $E D$

3. SNIH Saddle-node at invariant $S_{2}$ planes (inside tetrahedra) on the line $D L$

4. SCIH Saddle connection bifurcation at invariant $S_{2}$ planes in a small neighbourhood of the point $D$

5. PFH Pitchfork bifurcation at invariant $S_{2} \times S_{2}$ lines on the line $B V$.

Robust heteroclinic cycles exist within the region $B E D T L V$ in the parameter plane in Figure 5. These heteroclinic cycles are stable for $\alpha<\pi / 2$ and unstable when $\alpha>\pi / 2$ with a resonance bifurcation at $\alpha=\pi / 2$. For most of the region of parameters BEDTLM when heteroclinic cycles are the only attractors of the system (5), although near the line $T L$ there are also attracting solutions with symmetry $S_{2}$. Note that there exist two-dimensional sets of connecting orbits within these heteroclinic cycles when parameters $(\alpha, r)=(\pi / 2,0)$ or when we have $(\alpha, r)$ on the SNH line ED in Figure 5. In particular the bifurcations TCPFH and SNH give networks that, by Lemma 2 give extreme sensitivity; the other bifurcations to robust heteroclinic cycles give cycles that are contractible to the diagonal meaning we do not have extreme sensitivity in these cases.

\section{Discussion}

The paper presents a detailed bifurcation analysis of the system (2) for $N=3$ and $N=4$ oscillators on varying coupling parameters $\alpha, r$ plane and assuming assuming no detuning. We have identified what we believe to be all cases of bifurcation to dynamics that may give, on addition of detuning, extreme sensitivity. In all cases, these are associated with the creation of heteroclinic or homoclinic networks.

These bifurcation scenarios are surprisingly rich, given the small number of degrees of freedom and this richness is a consequence of the symmetries $S_{N}$ of the system. The symmetries give rise to codimension-one bifurcations with two or more dimensional centre manifolds and/or non-trivial constraints on the normal forms (see for example [12]), however the topology of the torus means that local bifurcations often have global consequences. There exist values of parameters $(\alpha, r)$ such that system (5) has two or more dimensional sets of heteroclinic cycles. For $N=3$ this is point $D$ in the parameter plane, while for $N=4$ it includes the line of the saddle-node/heteroclinic bifurcation on invariant lines $B E$ as well. 


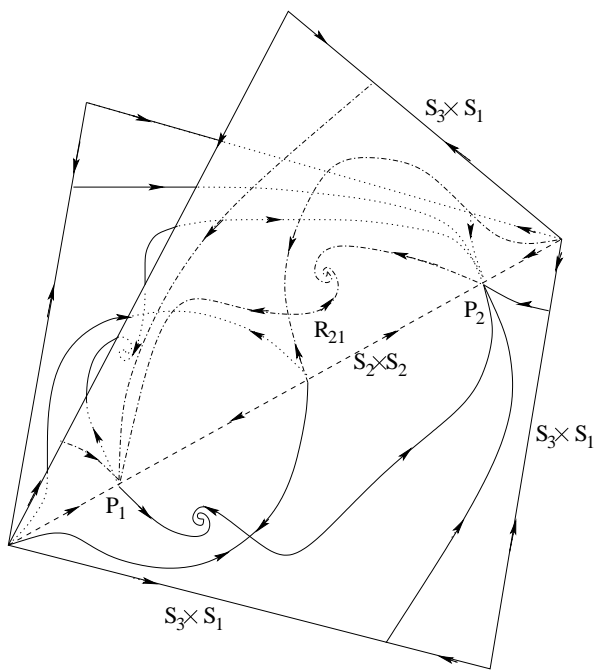

(a)

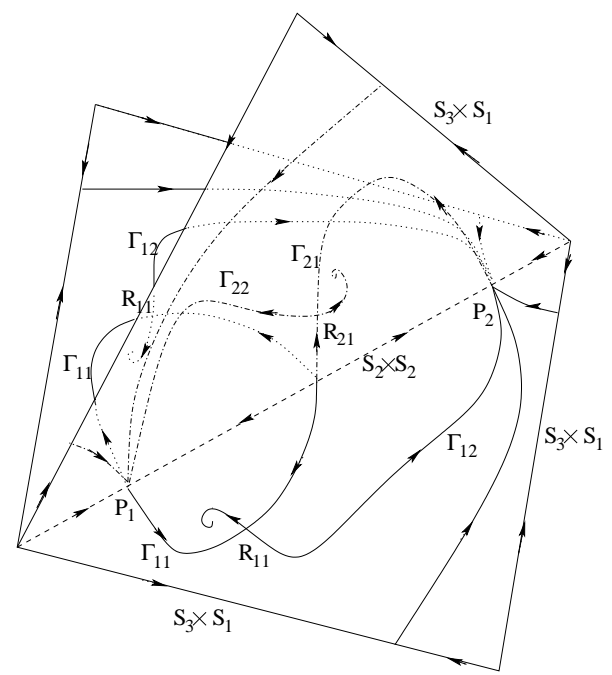

(b)

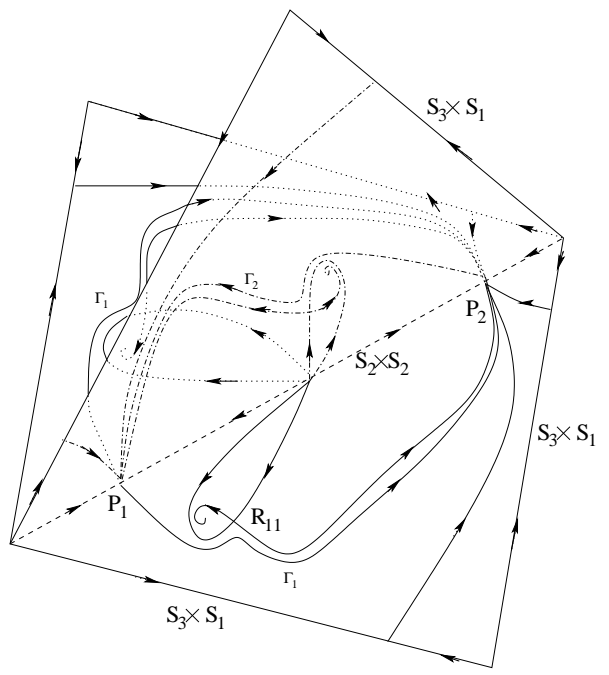

(c)

Figure 16: Schematic diagram showing situation (a) before (b) at and (c) after the heteroclinic bifurcation near the point $T$ in Figure 5 for $N=4$. Note that after the bifurcation there are heteroclinic cycles composed of two connecting orbits. 
Nonetheless, the bifurcations described are for the most part generic in the context of the symmetries present and hence are both robust and independent of exact choice of coupling function. Consideration of more general coupling functions can certainly give rise to dynamics that is not visible in (2); for example [6] consider a similar system of identical oscillators with an extra parameter $\beta, g(x)=\sin (x+\alpha)+r \sin (2 x+\beta)$, and find other nontrivial types of clustering appear for larger $N$ compared to the case $\beta=0$. They also find chaotic attractors in the case $N=5$ that we do not find for $N \leq 4$. We remark that the bifurcations detailed here should be observable (up to presence of perturbations) for more more general coupling functions; see e.g. [16].

\subsection{Results for higher numbers of oscillators}

The bifurcations of (5) for general $N$ are highly complicated, but we can characterise some properties that we now summarise. From [3] we have the following conclusions: The in-phase solution (origin) is an equilibrium state for all $r$ and $\alpha$. It is a sink for $\alpha \in(-\arccos (2 r), \arccos (2 r))$, a degenerate saddle when $\alpha= \pm \arccos (2 r)$, and a source for other $\alpha$. The system has an invariant manifold (4) for any $(r, \alpha)$. This manifold contains a point with $\mathbb{Z}_{N}$ symmetry that is always an equilibrium state. The system (5) has invariant subspaces of all dimensions between 1 and $N-2$ given by all possible clusterings of oscillators. At $r=\frac{1}{2} \cos \alpha$ the origin for (5) has a transcritical homoclinic bifurcation as long as $r \in(0, \tilde{r})$, where

$$
\tilde{r}=\frac{N-2}{2 \sqrt{2 N^{2}-4 N+4}} .
$$

This bifurcation is a transcritical-pitchfork/heteroclinic for even values of $N$. The hetero-

clinic connections consist of the $N$ invariant lines of form $S_{N-1} \times S_{1}$. System (5) has a saddle-node/heteroclinic bifurcation for $r \in[\tilde{r}, \bar{r}]$ for some $\bar{r}<0.5$. At the moment of intersection of saddle-node and transcritical bifurcation lines in the parameter plane, saddle-node points occur within each of the invariant $S_{N-1} \times S_{1}$ lines.

We note that robust heteroclinic cycles giving robust extreme sensitivity to detuning also appear and appear to be generic in cases where $N \geq 5$; see $[5,8,6]$. The structure of these networks of heteroclinic cycles can be highly nontrivial and is assoicated with a wide variety of bifurcations that we do not characterise here.

\section{Acknowledgements}

We thank the Royal Society for a visitor grant enabling OB to visit Exeter for March and April 2006. We also thank the EPSRC for partial support via EP/C510771 (PA) and the referees for their very helpful comments. 


\section{References}

[1] V. Afraimovich, M.I. Rabinovich, P. Varona, International Journal of Bifurcation and Chaos, 14: 1195-1208 (2004).

[2] E. Akin. The general topology of dynamical systems, AMS Graduate studies in Mathematics Volume 1, (1993).

[3] P. Ashwin and J.W. Swift, J. Nonlinear Sci. 2, 69-108 (1992).

[4] P. Ashwin, G.P. King and J.W. Swift, Nonlinearity, 4, 585-603 (1990).

[5] P. Ashwin, O. Burylko, Y. Maistrenko and O. Popovych. Phys Rev. Lett. 96, 05410 (2006).

[6] P. Ashwin, G. Orosz, J. Wordsworth and S.B. Townley. To appear in: SIAM J Appl. Dynamical Systems (2007)

[7] P. Ashwin and M. Timme. Nature 436, 36-37 (2005).

[8] P. Ashwin and J. Borresen. Phys. Rev. E 70, 026203 (2004).

[9] E. Brown, P. Holmes, and J. Moehlis. "Globally coupled oscillator networks" In: Perspectives and Problems in Nonlinear Science: A Celebratory Volume in Honor of Larry Sirovich, E. Kaplan, J. Marsden, K. Sreenivasan, Eds., p. 183-215. Springer, New York, (2003).

[10] E.J. Doedel, R.C. Poffenroth, A.R. Champneys, T.F. Fairgrieve, Yu.A. Kuznetsov, B.E. Oldeman, B. Sandstede, X. Wang, AUTO 2000: Continuation and bifurcation software for ordinary differential equations (With HomCont), User's guide, Concordia University (2006).

[11] G.B. Ermentrout, A Guide to XPPAUT for Researchers and Students, SIAM Publications (2002).

[12] M.Golubitsky and I. Stewart, The symmetry perspective. Birkäuser (2003).

[13] D. Hansel, G. Mato and C. Meunier, Phys. Rev. E 48, 3470-7 (1993).

[14] S.K. Han, C. Kurrer and Y. Kuramoto, Phys. Rev. Lett. 75, 3190-3 (1995).

[15] Y.B. Kazanovich and R.M. Borisyuk, Neural Networks 12, 441-453 (1999).

[16] I.Z. Kiss, C.G. Rusin, H. Kori and J.L. Hudson, Science 316, 1886-1889 (2007).

[17] H. Kori and Y. Kuramoto, Phys. Rev. E 63, 046214 (2001).

[18] H. Kori, Phys. Rev. E 68, 021919 (2003). 
[19] Y. Kuramoto, pp420-422 of Springer Lecture Notes in Physics Volume 39. Springer, Berlin (1975).

[20] Y. Kuramoto, Chemical Oscillations, Waves and Turbulence Springer-Verlag, Berlin, (1984).

[21] Yu. A. Kuznetsov, Elements of Applied Bifurcation Theory Springer-Verlag, New York, (2004).

[22] Y. Maistrenko, O. Popovych, O. Burylko and P.A. Tass, Phys. Rev. Lett. 93, 084102 (2004).

[23] H. Sakaguchi and Y. Kuramoto, Progress of Theoretical Physics 76, 576-581 (1986).

[24] S.H. Strogatz, Physica D 143, 1-20 (2000).

[25] L.S. Tsimring, N.F. Rulkov, M.L. Larsen and M. Gabbay, Phys. Rev. Lett. 95, 014101 (2005).

[26] E.A. Viktorov, A.G. Vladimirov and P. Mandel, Phys. Rev. E 62, 6312-7 (2000).

[27] S. Watanube and S.H. Strogatz, Physica D 74, 197-253 (1994).

[28] K. Wiesenfeld, P. Colet, and S. H. Strogatz, Phys. Rev. E 57, 1563-9 (1998).

[29] A. Winfree, The geometry of biological time Springer, New York (2001). 


\section{A Structure of the set $M^{(N)}$}

Recall that

$$
M^{(N)}=\left\{\left(\theta_{1}, \cdots, \theta_{N}\right): \sum_{j=1}^{N} e^{i \theta_{j}}=0\right\} .
$$

We claim that the set $M^{(N)}$ has dimension $N-2$ for $N \geq 3$. To see this, take a typical point in the set and note that without loss of generality we can assume the $\theta_{i} \in[0,2 \pi)$ are sorted into increasing order. Consider each $e^{i \theta_{i}}$ as a unit vector in the plane; if we write

$$
A_{k}=\sum_{j=1}^{k} e^{i \theta_{j}}
$$

then the points $A_{k}$ represent vertices of a convex polygon with unit-length edges; i.e. such that $\left|A_{k+1}-A_{k}\right|=1, k=1, \cdots N-1$ and $A_{N}=0$. Note that the complex constraint $A_{N}=0$ restricts two degrees of freedom in choice of the $N$ angles $\theta_{i}$ and hence there are $N-2$ degrees of freedom remaining in choice of $\theta_{i}$.

For $N=3$ the polygons represented in this way are precisely the equilateral triangles with unit side-length and one corner at the origin. For $N=4$ the polygons are rhombi with unit side-length and one corner at the origin. 